\title{
Effects of Monolayer Density and Bulk Ionic Strength on Acid-Base Equilibria at the Air/Water Interface
}

\author{
Hongfei Wang, ${ }^{\dagger}$ Xiaolin Zhao, and K. B. Eisenthal* \\ Department of Chemistry, Columbia University, New York, New York 10027
}

Received: May 17, 2000; In Final Form: July 18, 2000

\begin{abstract}
The surface density and electrolyte concentration dependences of the $\mathrm{p} K_{\mathrm{a}}$ of $\mathrm{CH}_{3}\left(\mathrm{CH}_{2}\right)_{21} \mathrm{NH}_{3}{ }^{+}$at the air/ water interface are obtained using the method of second harmonic generation. If it is assumed that the amphiphiles are uniformly distributed at the interface, the application of the Gouy-Chapman model yields $\mathrm{p} K_{\mathrm{a}}$ values ranging from 10.2 to 8.2 as the interface density varies from 100 to $22 \AA^{2} /$ molecule. By accounting for the inhomogeneity of the interface, i.e., there are amphiphiles islands of "liquid phase" density immersed in a "gas phase" of very low-density amphiphiles, a single $\mathrm{p} K_{\mathrm{a}}$ of $10.1 \pm 0.2$ is obtained at all interface densities. The acidity of the long chain amine is enhanced by 3 times at the air/water interface compared with the bulk aqueous solution.
\end{abstract}

\section{Introduction}

The special chemical and physical properties of interfaces are due to the asymmetry in forces that molecules at the interface experience. The bulk media that define a given interface are themselves markedly different from each other in their properties as exemplified by the vapor/liquid, nonpolar liquid/polar liquid and liquid/solid interfaces. The unique environment of the interface, which differentiates it from the bulk media, is seen in many of its time independent properties such as chemical equilibria, composition, and $\mathrm{pH}$, as well as in such time dependent properties as molecular motions, every relaxation and chemical reactions. ${ }^{1,2}$ A powerful way to selectively probe interfaces and avoid the overwhelming contributions of the far larger bulk media to the probe signal is the use of the nonlinear optical methods of second harmonic (SHG) and sum frequency generation (SFG). ${ }^{3,4}$ The key feature of these spectroscopic methods is their inherent sensitivity to the noncentrosymmetric interface located between two centrosymmetric media. ${ }^{5-8}$

\section{Measurement of Interface Acid-Base Equilibria}

Second harmonic generation has been used in essentially two different ways to determine the $\mathrm{p} K_{\mathrm{a}}$ values of interface species. ${ }^{9,13}$ The different approaches are applicable to distinctly different physical situations. In one of the methods the relative populations of the acid and base forms of the chemical species at the interface are obtained from measurements of the SHG signal as a function of the bulk $\mathrm{pH}_{\mathrm{b}} \cdot{ }^{9,10}$ The interface $\mathrm{pH}_{\mathrm{s}}$ is obtained by combining the interface charge density, which is determined by the charged acid or base forms, with the GuoyChapman model of the charged interface. The relation between these various quantities is given by

$$
\mathrm{p} K_{\mathrm{a} \mathrm{s}}=\mathrm{pH}_{\mathrm{s}}+\log \frac{\left[\mathrm{HA}^{+}\right]_{\mathrm{s}}}{[\mathrm{A}]_{\mathrm{s}}}
$$

$\dagger$ Molecular Reaction Dynamics Laboratory, Institute of Chemistry, Chinese Academy of Sciences, Beijing, 100080, P. R. China. E-mail: hongfei@mrdlab.icas.ac.cn. where $[\mathrm{HA}]_{\mathrm{s}}$ and $[\mathrm{A}]_{\mathrm{S}}$ are the interface populations of the acid and base forms, respectively. The physical situation to which this approach is applicable and has proven successful requires that the second-order nonlinearity $\chi^{(2)}$ of the acid-base forms be significantly larger than the $\chi^{(2)}$ of the background water or other interface species. For the aromatic acids, such as anilinium $\left(\mathrm{C}_{6} \mathrm{H}_{5} \mathrm{NH}_{3}{ }^{+}\right)$and phenol $\left(\mathrm{C}_{6} \mathrm{H}_{5} \mathrm{OH}\right)$, their large second-order nonlinearities yield second harmonic signals significantly above that of interfacial water. However, for saturated organic and inorganic acids the magnitude of their $\chi^{(2)}$ values are typically less than or comparable to the $\chi^{(2)}$ of water. As a consequence, the aforementioned second harmonic method is difficult to apply.

It is for this latter case of interfacial acid/bases that have relatively small $\chi^{(2)}$ values, where a different second harmonic method becomes useful. This other method for determining the $\mathrm{p} K_{\mathrm{a}}$ of interfacial acids is based on the finding ${ }^{11,12}$ that an interface consisting of charged species polarizes the bulk water molecules and thereby generates a significant SH signal by a third-order process characterized by the nonlinear susceptibility $\chi^{(3)}$. It has been found that the magnitude of the $\mathrm{SH}$ signal from the $\chi^{(3)}$ process for systems such as the solid silica $(-\mathrm{SiOH}) /$ aqueous interface ${ }^{11}$ as well as negatively and positively charged monolayers at air/aqueous interfaces, ${ }^{12}$ is comparable to or larger than the $\chi^{(2)}$ contributions from the background water and other interfacial species.

For a charged interface, the total SH electric field, which is the square root of the SH intensity signal, can be expressed as ${ }^{13}$

$$
E_{2 \omega}=A+B \Phi(0)
$$

where $A$ is the total effective $\chi^{(2)}$ contribution from the water and the acid and base species at the interface, $B$ is the effective $\chi^{(3)}$ due to the polarized water, which extends into the bulk solution, and $\Phi(0)$ is the electric potential of the charged plane at the interface. It is to be noted that the relative phase of $A$ and $B \Phi(0)$ terms affects the magnitude of $E_{2 \omega}$. For nonresonant cases, the relative phase can be either $0^{\circ}$ or $180^{\circ}$ only; i.e., the relative sign of $A$ and $B \Phi(0)$ can be either +1 or -1 . In the work reported here the $\mathrm{SH}$ field $E_{2 \omega}$ is a normalized quantity 
having been divided by the $\mathrm{SH}$ field obtained from the neat air/water interface. The SH field is linearly proportional to the interfacial potential $\Phi(0)$, as shown in eq 1 with a proportionality constant $B$, which was found to have the same value for the several aqueous interfaces that have been studied. ${ }^{12,13}$ This finding that the value of $B$ is the same from the silica/aqueous interface, the positively charged $\mathrm{CH}_{3}\left(\mathrm{CH}_{2}\right)_{2} \mathrm{~N}^{+}\left(\mathrm{CH}_{3}\right)_{3}$ monolayer at the air/aqueous interface, and the negatively charged $\mathrm{CH}_{3}\left(\mathrm{CH}_{2}\right)_{21} \mathrm{SO}_{3}{ }^{-}$monolayer at the air/aqueous interface indicates that $B$ depends chiefly on the bulk liquid, which in these experiments is water. From eq 1 we see that the interface potential can be obtained from determination of the value of $A$, use of the water constant $B$, and measurement of $E_{2 \omega}$. This approach provides a different way to measure interface $\mathrm{p} K_{\mathrm{a}}$ values as described in the following discussion.

For the acid-base reaction

$$
\mathrm{HA}^{+}+\mathrm{H}_{2} \mathrm{O} \rightleftharpoons \mathrm{A}+\mathrm{H}_{3} \mathrm{O}^{+}
$$

the surface $K_{\mathrm{a}}$ can be written as

$$
K_{\mathrm{a}}=\frac{f}{1-f}\left(\mathrm{H}_{3} \mathrm{O}^{+}\right)_{\mathrm{s}}=\frac{f}{1-f}\left(\mathrm{H}_{3} \mathrm{O}^{+}\right)_{\mathrm{b}} \exp \frac{-e \Phi(0)}{k T}
$$

where $f$ is the molar fraction of the neutral form $\mathrm{A}$ at the interface, $\left[\mathrm{H}_{3} \mathrm{O}^{+}\right]_{\mathrm{S}}$ is the interface hydronium ion concentration, $\left[\mathrm{H}_{3} \mathrm{O}^{+}\right]_{\mathrm{b}}$ is the bulk hydronium ion concentration, and $\Phi(0)$ is the electric potential at the charge plane. From eq 3 we see that, to obtain the surface $\mathrm{p} K_{\mathrm{a}}$, it is necessary to measure $f$ and $\Phi(0)$. According to eq 1 , we can use SHG to measure $\Phi(0)$ if $A$ and $B$ can be evaluated. Because the $B$ value for aqueous interfaces $^{11,12}$ is known, we have to determine only $A$ and $\Phi(0)$. The $A$ term is the $\chi^{(2)}$ contribution of all interfacial species, which can be expressed as

$$
\begin{aligned}
\mathrm{A} & =(1-f) A_{\text {acid }}+f A_{\text {base }}+A_{\text {water }} \\
& =(1-f)\left(A_{\text {acid }}+A_{\text {water }}\right)+f\left(A_{\text {base }}+A_{\text {water }}\right)
\end{aligned}
$$

where $A_{\text {water }}, A_{\text {acid }}$, and $A_{\text {base }}$ are the effective $\chi^{(2)}$ values of water, acid, and base species, respectively. The summed quantities $A_{\text {acid }}+A_{\text {water }}$ and $A_{\text {base }}+A_{\text {water }}$ are obtained from $\mathrm{SH}$ measurements on the completely charged monolayer $(f=0)$ and the neutral monolayer $(f=1)$, respectively. From eq 4 we see that $A$ can then be expressed in terms of the single unknown quantity $f$, which is related to $K_{\mathrm{a}}$ by eq 3 . In this way it is possible to fit the $E_{2 \omega}$ vs bulk pH curve with the surface $\mathrm{p} K_{\mathrm{a}}$ as the fitting parameter. It is interesting to notice that for the nonresonant monolayers studied the total $\chi^{(2)}$ is comparable to that of neat water, thus indicating a dominant contribution of water molecules to $\chi^{(2)}$. We have seen that this is the case for the positively charged monolayer $\mathrm{CH}_{3}\left(\mathrm{CH}_{2}\right)_{21} \mathrm{~N}^{+}\left(\mathrm{CH}_{3}\right)_{3}$ and the negatively charged monolayer $\mathrm{CH}_{3}\left(\mathrm{CH}_{2}\right)_{21} \mathrm{SO}_{3}{ }^{-}$at air/water interfaces. $^{12}$

Experiments on charged air/aqueous interfaces have shown that the Guoy-Chapman electric double-layer model describing

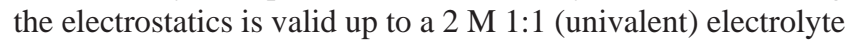
concentration. ${ }^{14}$ For this model, the electric potential at the charge plane, $\Phi(0)$, is related to the interfacial charge density and total electrolyte concentration:

$$
\Phi(0)=\frac{2 k T}{z e} \sinh ^{-1}\left(\sigma \sqrt{\frac{\pi}{2 C \epsilon k T}}\right)
$$

where $\sigma$ is the charge density at the interface, $e$ is the electronic charge, $\epsilon$ is the bulk dielectric constant, $C$ is the total bulk electrolyte concentration in units of moles of ions $/ \mathrm{cm}^{3}$, and $z$ is the sign of the charged monolayer. In the case of an ionizable $\mathrm{HA}^{+}$insoluble monolayer, the surface charge density $\sigma$ is directly related to the degree of ionization $f$, by $\sigma=(1-f) \sigma_{\mathrm{m}}$, where $\sigma_{\mathrm{m}}$ is the surface charge when the monolayer is completely ionized. $\sigma_{\mathrm{m}}$ is known for a given spread monolayer density. Therefore, $\Phi(0)$ is only a function of $f$, and the total SH field is only a function of $f$. The determination of $\Phi(0)$ from $\mathrm{SH}$ measurements is used to obtain the ionization degree $f$ from eq 5 . The $\mathrm{p} K_{\mathrm{a}}$ is then readily obtained by using eq 3 . This method for surface $\mathrm{p} K_{\mathrm{a}}$ determination is referred to as the $\chi^{(3)}$ method. ${ }^{13}$

\section{Experimental Section}

The SHG experimental setup and the sample preparation has been described in previous papers. ${ }^{11-13}$ Two different laser systems were used in the reported experiments: (a) $\mathrm{Ar}^{+}$ion laser sync-pumped and cavity-dumped picosecond dye laser system and (b) $\mathrm{Ar}^{+}$ion laser-pumped femtosecond Ti:sapphire laser system. The dye laser system operated at $610 \mathrm{~nm}$ with a pulse width of $5 \mathrm{ps}$ and a repetition rate of $4.1 \mathrm{MHz}$ and the Ti:sapphire laser was operating at $810 \mathrm{~nm}$ with a pulse width of $100 \mathrm{fs}$ and a repetition rate of $82 \mathrm{MHz}$. The latter system gives a much larger signal and better signal-to-noise ratio.

The docosylamine $\mathrm{CH}_{3}\left(\mathrm{CH}_{2}\right)_{21} \mathrm{NH}_{2}$ molecule noted as $\mathrm{C}_{22}$ $\mathrm{NH}_{2}$ or $\mathrm{C}_{22}$ amine, was synthesized from $\mathrm{CH}_{3}\left(\mathrm{CH}_{2}\right)_{20} \mathrm{CN}$ by reduction with $\mathrm{LiAlH}_{4}$ in anhydrous ether. The product was then passed through a silica column, and the main component was identified using mass spectra and NMR. It was then recrystallized from ethanol at least four times before being used. The purity of the sample was better than $99 \%$. In the experiments where the surface density was varied, the $\mathrm{C}_{22} \mathrm{NH}_{2} /$ hexane solution was spread at a series of areas per molecule, 100, 70, $50,40,30,25$ and $22 \AA^{2}$ on an aqueous solution containing $\mathrm{KCl}, \mathrm{KOH}$, and $\mathrm{HCl}$ with a fixed total electrolyte concentration of $10 \mathrm{mM}$. In the experiments where the bulk electrolyte concentration was varied, the $\mathrm{C}_{22} \mathrm{NH}_{2}$ /hexane solution was spread at a density of $50 \AA^{2}$ per molecule on an aqueous solution containing $\mathrm{KCl}, \mathrm{KOH}$, and $\mathrm{HCl}$ at 100,10 , and $1 \mathrm{mM}$ bulk electrolyte concentrations. The $\mathrm{pH}$ of the aqueous solution was measured with a Corning $\mathrm{pH}$ meter. The $\mathrm{SH}$ signal from the sample was normalized to the $\mathrm{SH}$ signal of the neat air/water interface, which was found to be independent of bulk $\mathrm{pH}$. The $\pi-A$ curves were measured with an electronic balance in a homemade Teflon Langmuir trough. Each point was measured $10 \mathrm{~s}$ after a slow compression.

\section{Results and Discussion}

4.1. Dependence of $\mathrm{p} K_{\mathrm{a}}$ on Amphiphile Surface Density. Figure 1 shows the normalized SH electric field generated by the ionizable monolayer $\mathrm{C}_{22}$ amine as a function of the bulk $\mathrm{pH}$ at surface densities of $100,70,50,40,30,25$, and $22 \AA^{2}$ per molecule and a total electrolyte concentration of $10 \mathrm{mM}$. We obtained data for densities of 100 , 50, and $40 \AA^{2}$ per molecule with the dye laser system at $610 \mathrm{~nm}$ (SHG at 305 $\mathrm{nm})$ and $70,50,40,30,25$, and $22 \AA^{2}$ per molecule with the Ti:sapphire system at $810 \mathrm{~nm}$ (SHG at $405 \mathrm{~nm}$ ). Figure 2 shows the comparison of the data from the two laser systems for surface densities of 50 and $40 \AA^{2}$ per molecule. From Figure 2 we can see that the $\mathrm{SH}$ signal is independent of the wavelength of the incident laser light. This is consistent with the nonresonant nature of the $\mathrm{SH}$ signal for the charged interfacial systems studied. 


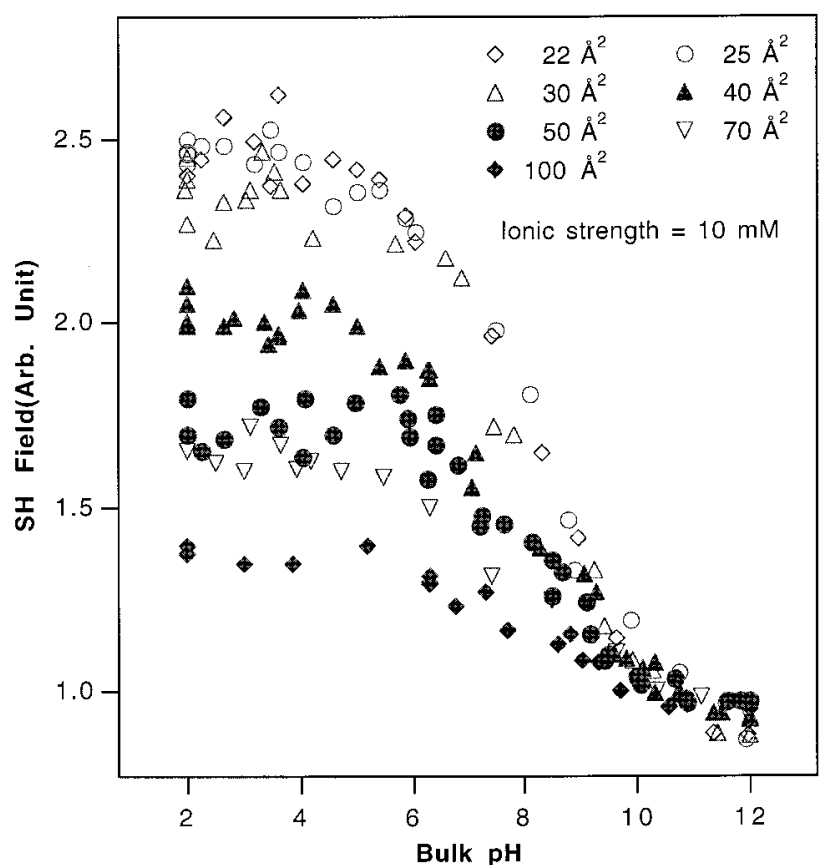

Figure 1. Complete data for different surface densities. The trend of density and $\mathrm{pH}$ dependence is clearly shown.
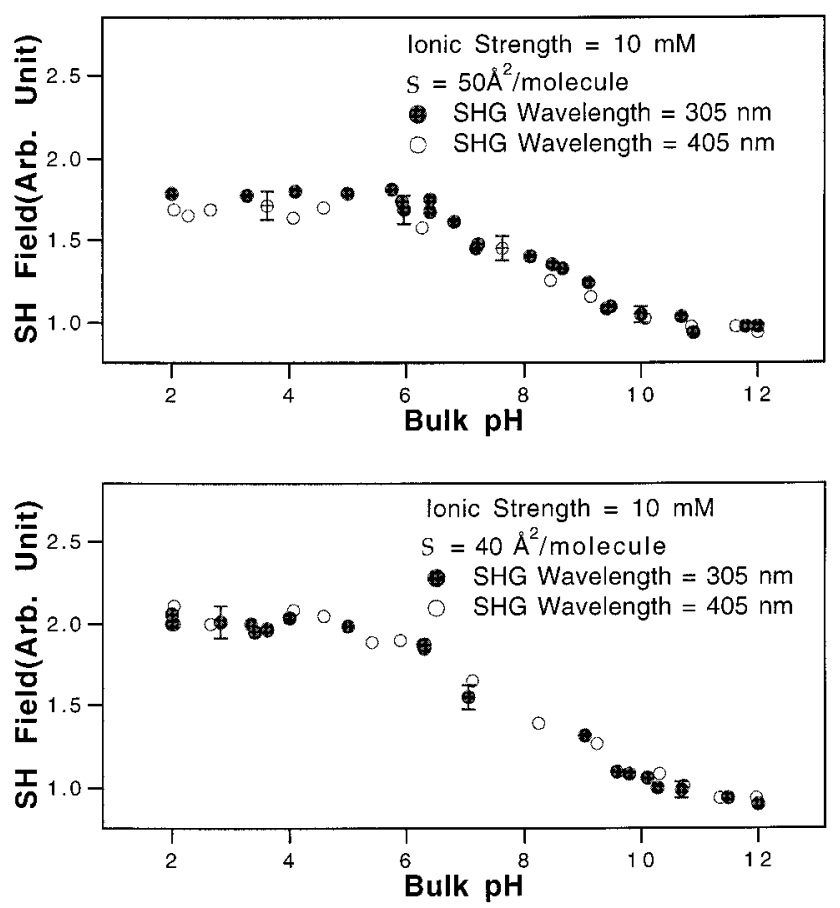

Figure 2. Comparison of the data taken at different $\mathrm{SH}$ wavelengths. The good agreement shows the nonresonant nature of $\mathrm{SH}$ generation.

There are two general trends displayed in the data of Figure 1. One is that the $\mathrm{SH}$ electric field increases as the bulk $\mathrm{pH}$ decreases and, second, that the $\mathrm{SH}$ field increases as the surface density increases. As the bulk $\mathrm{pH}$ decreases, the ionizable monolayer $\mathrm{C}_{22}$ amine becomes progressively charged, which therefore increases the surface potential. It is inferred from Figure 1 that the signs of the $A$ and $B \Phi$ terms appearing in eq 1 , are the same because the $\mathrm{SH}$ signal is seen to increase as the potential increases, i.e., as the bulk $\mathrm{pH}$ decreases. In addition, it is to be noted that the $\mathrm{SH}$ field is expected to increase with surface density because the SH field is proportional to the susceptibility $\chi^{(2)}$, which in turn is proportional to the surface density. This trend is also seen in Figure 1.

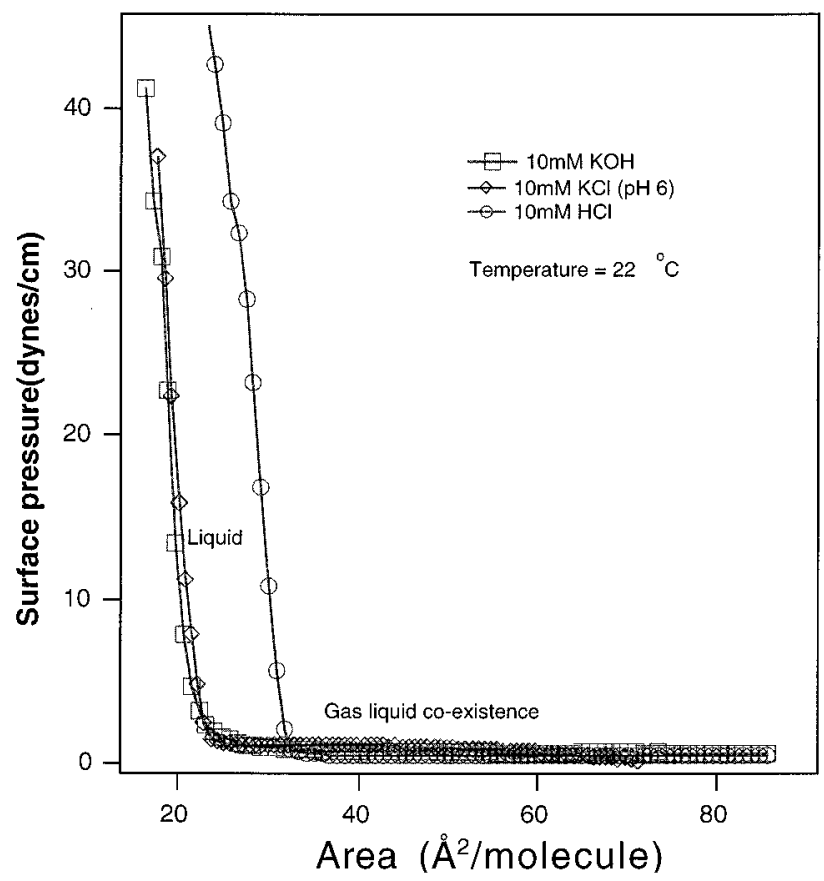

Figure 3. Phase Diagram of $\mathrm{C}_{22}$ amine and its charged form. The expansion of the liquid phase due to charged form is clearly seen.

We also found that each one of these curves in Figure 1 looks like a titration curve. We applied the $\chi^{(3)}$ method to our data, with $1 / \sigma_{\mathrm{m}}=100,50,40,30,25$, and $22 \AA^{2}$ per molecule, respectively, and obtained a series of different surface $\mathrm{p} K_{\mathrm{a}}$ values ranging from $8.2,8.9,9.4,9.9,10.1$, to $10.2 \pm 0.2$ for the area/ molecule from 22 to $100 \AA^{2}$.

In applying the $\chi^{(3)}$ method to obtain these $\mathrm{p} K_{\mathrm{a}}$ values, the implicit assumption is made that the surface was homogeneous, i.e., that the surface charges due to the acidic form $\mathrm{CH}_{3}\left(\mathrm{CH}_{2}\right)_{19^{-}}$ $\mathrm{NH}_{3}{ }^{+}$are uniformly distributed on the aqueous interface. Figure 3 shows the phase diagrams (surface pressure $\pi$ vs $S$, the reciprocal of surface density) of $\mathrm{C}_{22}$ amine on the surface of 10 $\mathrm{mM}$ solutions with three bulk $\mathrm{pH}$ values: 12.0 for $10 \mathrm{mM} \mathrm{KOH}$, 6.0 for $10 \mathrm{mM} \mathrm{KCl}$ and 2.0 for $10 \mathrm{mM} \mathrm{HCl}$, respectively. A surface diagram is the 2-D analogue of a 3-D pressure-volume phase diagram, which gives information on the various monolayer states. ${ }^{16 a, b}$ For a single $\pi-S$, curve the flat part is called the gas-liquid coexistence region. To the left of it is called the liquid or condensed region while to the right is the gas region, which is not shown in the phase diagram since it is far to the right. The turning point from the gas-liquid coexistence region to the gas phase region is greater than $400 \AA^{2}$ for the $C_{22}$ amine. As in the 3-D system, there are three surface states to consider: (a) when the total surface density is in the liquid region of the phase diagram, which is at areas/molecule less than the coexistence-liquid transition density, $S_{1}$, the monolayer molecules are uniformly distributed on the surface with a relatively high density which is the same as the total surface density; (b) when the total surface density is in the coexistence region, rather than being uniformly distributed, the monolayer molecules are distributed into distinct regions. In some regions of the surface there are liquid islands that have the density equal to the density at the gas-liquid to liquid region turning point $\left(S_{1}\right)$. The other regions are gas phase (low density) regions consisting of isolated amphiphilic molecules, which separate the liquid density islands and have a gas phase density of the gas-liquid to gas turning point $\left(S_{\mathrm{g}}\right)$. With the knowledge of $S_{\mathrm{l}}$ and $S_{\mathrm{g}}$, we can use the thermodynamic lever rule to calculate the area fraction of both phases for a given total surface density. When the total surface 
density is in the gas region (very low density) of the phase diagram, the monolayer molecules are on average very far apart from each other. In the coexistence region the surface is inhomogeneous and can be represented schematically as

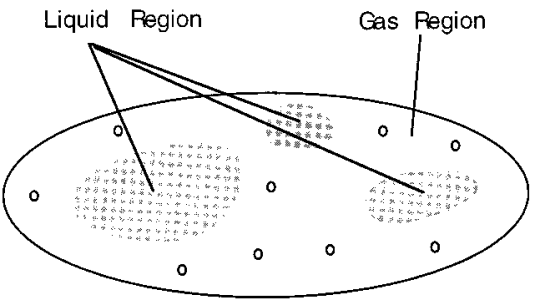

In the $\chi^{(3)}$ method just discussed we applied the GuoyChapman model to a surface assumed to be homogeneous. This approach is not directly applicable to the nonuniform surface schematically shown above. We now discuss application of the $\chi^{(3)}$ method to the physically realistic inhomogeneous surface when the system is at a density corresponding to the coexistence region. The SH signal is obtained upon irradiating the interface with a large area laser beam. In this way both the high-density liquid island regions and the low-density gas phase regions of the interface are exposed to the incident laser beam. Let $I$ be the measured SH signal, $I_{1}$ and $I_{\mathrm{g}}$ be the signal if the surface is covered by a homogeneous layer of liquid (l) or gas (g), and $X_{1}$ and $1-X_{1}$ be the area fraction of liquid and gas phase for a given total surface density, respectively. We can then write

$$
I=I_{1} X_{1}+I_{\mathrm{g}}\left(1-X_{1}\right)
$$

and

$$
I_{1}=\frac{I-\left(1-X_{1}\right) I_{\mathrm{g}}}{X_{1}}=\frac{I-\left(1-X_{1}\right)}{X_{1}}
$$

with $I_{\mathrm{g}}=1$. Because the surface density of gas phase molecules is very low, being at least 20 times lower than that of liquid phase, we experimentally found in this nonresonant case that the gas phase signal $I_{\mathrm{g}}$ can be set within our experimental limits equal to the neat air/water surface signal $I_{\text {water }}$. Thus, our normalization of all signals to the neat air/water surface yields a value $I_{\mathrm{g}}=1$.

We define a reciprocal surface density $S$, area/molecule, which we know for the prepared sample. The lever rule gives

$$
X_{1}=\frac{S_{1}\left(S_{\mathrm{g}}-S\right)}{S\left(S_{\mathrm{g}}-S_{1}\right)}
$$

Combining $X_{1}$ obtained from eq 8 for the particular system under study, with the measured $\mathrm{SH}$ signal $I$, we can calculate with eq $7, I_{1}$, the signal for the homogeneous liquid phase.

With our surface pressure apparatus we cannot measure the exact value of the coexistence to gas turning point $S_{\mathrm{g}}$, because the surface pressure $(\pi)$ for the coexistence region is extremely small. ${ }^{15 a}$ It is generally believed that the $S_{\mathrm{g}}$ value is larger than several hundred or more than a thousand $\AA^{2} /$ molecule. ${ }^{15} \mathrm{We}$ can estimate a lower limit for $S_{\mathrm{g}}$ assuming an ideal 2-D gas equation, ${ }^{15 \mathrm{a}}$

$$
\pi S=k T
$$

where $\pi$ is in dynes/cm and $S$ is in $\AA^{2} /$ molecule. At room temperature, $T=294 \mathrm{~K}$, their product should be 406.2 $\left(\mathrm{erg} \cdot \AA^{2} \cdot \mathrm{cm}^{-2} \cdot\right.$ molecule $\left.{ }^{-1}\right)$. Since the surface pressure for the coexistence region (flat region) is less than $0.5 \mathrm{dyn} / \mathrm{cm}$, the $S_{\mathrm{g}}$
TABLE 1: Fitting Parameters and Results with $S_{\mathrm{l}}=$ $25 \AA^{2} /$ molecule

\begin{tabular}{ccccc}
\hline \multicolumn{2}{c}{$S_{1}=25 \AA^{2} /$ molecule } & & \multicolumn{2}{c}{$S_{\mathrm{g}}=800 \AA^{2} /$ molecule } \\
\cline { 1 - 2 }$S\left(\AA^{2} /\right.$ molecule $)$ & $X_{1}$ & & $1 / \sigma_{\mathrm{m}}\left(\AA^{2} /\right.$ molecule $)$ & surface $\mathrm{p} K_{\mathrm{a}}$ \\
\hline 100 & 0.226 & & 25 & $10.1 \pm 0.2$ \\
70 & 0.336 & & 25 & $10.0 \pm 0.2$ \\
50 & 0.484 & & 25 & $10.1 \pm 0.2$ \\
40 & 0.613 & & 25 & $10.3 \pm 0.2$ \\
30 & $0.828^{a}$ & & 25 & $10.0 \pm 0.2$ \\
25 & 1.000 & & 25 & $10.1 \pm 0.2$ \\
22 & 1.000 & 22 & $10.2 \pm 0.2$
\end{tabular}

${ }^{a}$ We used $X_{1}=1.000$ for the flat part at low $\mathrm{pH}$ region since $S_{1}=$ $32 \AA^{2} /$ molecule when totally charged.

value must be greater than 406.2/0.5 = 812.4 $\AA^{2} /$ molecule. Because $S_{\mathrm{g}}$ is very large compared with $S$ and $S_{\mathrm{l}}$, it follows that $\left(S_{\mathrm{g}}-\mathrm{S}\right) /\left(S_{\mathrm{g}}-S_{\mathrm{l}}\right)$ is very close to unity. For convenience, we select $S_{\mathrm{g}}=800 \AA^{2} /$ molecule as the lower limit in our calculation. We see from eq 8 that the $X_{1}$ value is not sensitive to such a large $S_{\mathrm{g}}$ value and is even less sensitive to larger values of $S_{\mathrm{g}}$.

From Figure 3 we see that the gas-liquid coexistence to liquid phase turning points $S_{1}$ are not the same for different $\mathrm{pH}$ 's. The turning point moves to lower density as the bulk $\mathrm{pH}$ is lowered (remember the higher the $S$ value, the lower the surface density). This is easily understood upon noting that at lower bulk $\mathrm{pH}$ value the $\mathrm{C}_{22}$ amine molecules are more charged and thus the liquid phase expands to a lower surface density because of electrostatic repulsions. Because $S_{1}$ changes with bulk $\mathrm{pH}$, we have to obtain phase diagrams for all $\mathrm{pH}$ values to get the complete set of $S_{1}$ values. We can then calculate the area fraction $X_{1}$ for all the total surface densities and all the $\mathrm{pH}$ values. Fortunately, we do not have to do this because we found that for a very large part of the $\mathrm{pH}$ regions, e.g., from $\mathrm{pH}=12.0$ to 6.04 , the turning point $S_{1}$ is about $25 \AA^{2} /$ molecule. This observation results from the fact that in this $\mathrm{pH}$ region the monolayer molecules are not significantly protonated and thus not significantly charged. It then follows that the expansion of the liquid phase is not significant and $S_{1}$ remains at the same value. The turning point for $\mathrm{pH}=2.0$ is about $32 \AA^{2} /$ molecule, while the turning points for $\mathrm{pH}=12.0$ and 6.04 are nearly the same at $25 \AA^{2} /$ molecule. For bulk pH's between 2.0 and 6.4 , the $S_{1}$ values are between the two values of 32 and $25 \AA^{2}$. To estimate how sensitive the area fraction $X_{1}$ was to the value of the turning point $S_{1}$, we calculated the $X_{1}$ values for an $S_{1}=25$ $\AA^{2}$ and $S_{1}=32 \AA^{2}$. Using these calculated $X_{1}$ values we then applied the $\chi^{(3)}$ method (let $1 / \sigma_{\mathrm{m}}=S_{\mathrm{l}}$ ) to fit the calculated liquid phase electric field $\sqrt{I_{1}}$ vs bulk $\mathrm{pH}$ data. We found that (a) for either $S_{1}$ value we used, the calculated $I_{1}$ values turned out to be very close to each other for all surface densities ranging from $100,70,50,40$ to $30 \AA^{2} /$ molecule and (b) for all the above densities, the surface $\mathrm{p} K_{\mathrm{a}}$ value we obtained by using $S_{\mathrm{l}}=25$ $\AA^{2} /$ molecule was $10.1 \pm 0.2$, while using $S_{1}=32 \AA^{2} /$ molecule we obtained $10.1 \pm 0.6$.

The parameters and fitting results for $S_{1}=25 \AA^{2} /$ molecule are in Table 1. Using the parameters in Table 1, the calculated $\mathrm{SH}$ electric field $\sqrt{I_{1}}$ and fitting are shown in Figure 4.

We note that by including the inhomogeneity of the surface in the coexistence region, we have succeeded in accounting for the apparently large differences (Figure 1 ) in surface $\mathrm{p} K_{\mathrm{a}}$ values of $8.2-10.2$, as the total surface density changed from 22 to $100 \AA^{2}$ (Figure 4). For experiments at densities of 25 and 22 $\AA^{2} /$ molecule we can directly apply the $\chi^{(3)}$ method, because the surface is not in the inhomogeneous coexistence region for these densities but rather is in the homogeneous liquid phase for these 


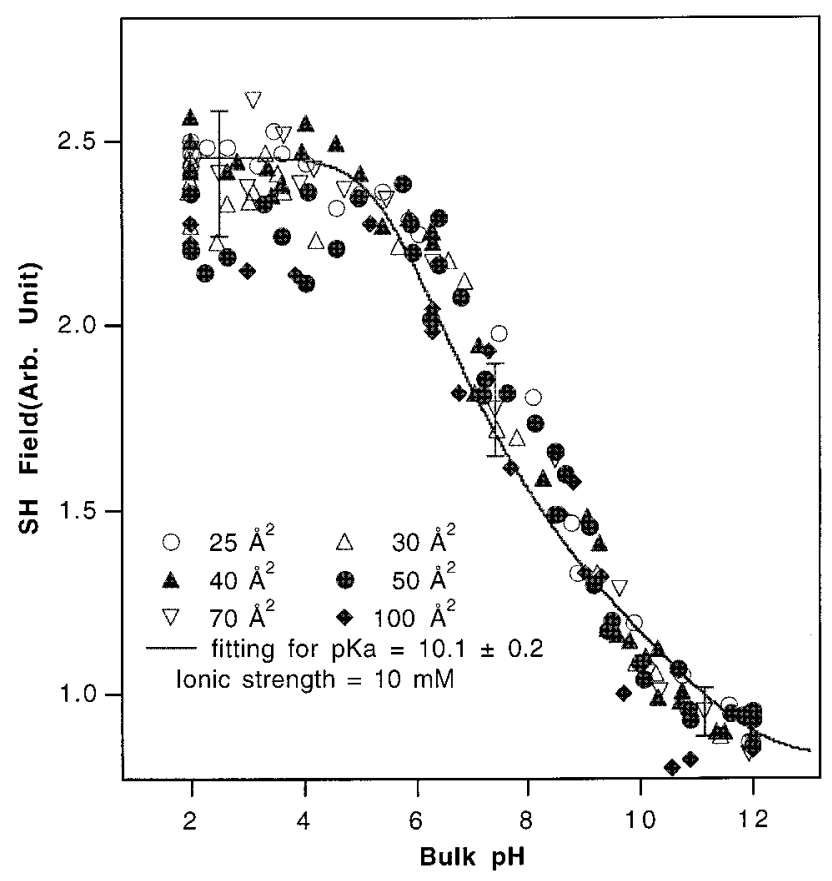

Figure 4. Calculated $\sqrt{I_{1}}$ and fitting(solid line). The $\sqrt{I_{1}}$, s calculated from different surface densities are close to each other. We should note that the calculation makes the small error of the experimental data bigger for calculated $\sqrt{I_{1}} . S_{1}$ and $X_{1}$ are listed in Table. 1. The curve was fitted using eqs 1,3 , and 4 .
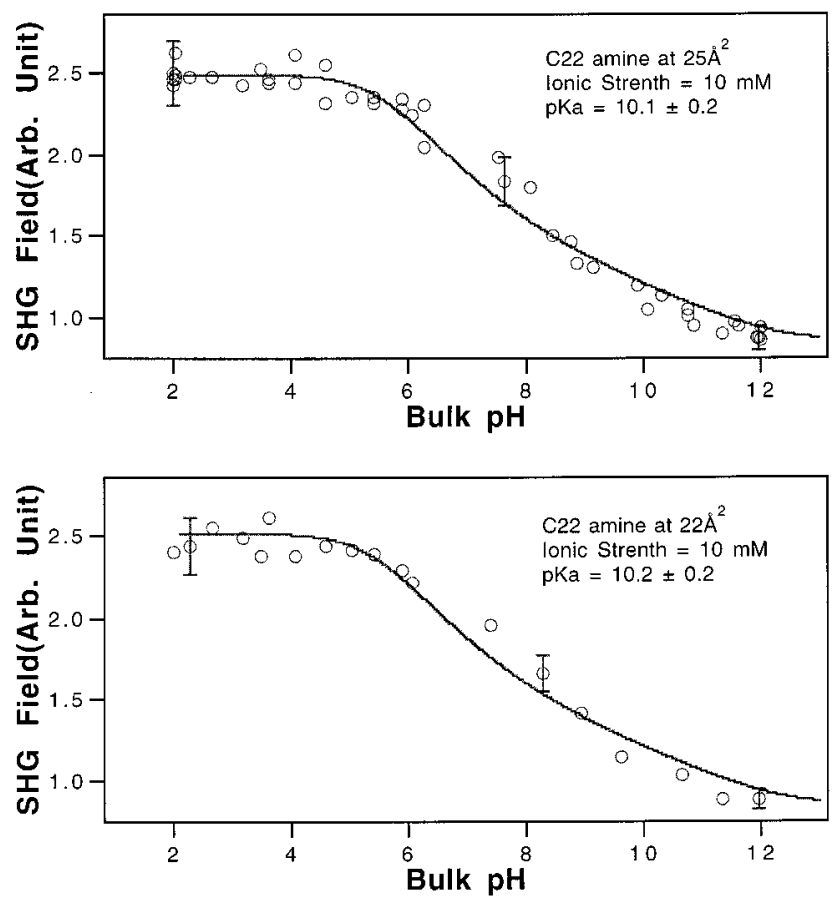

Figure 5. Data and fitting (solid line) for total surface densities of 25 and $2 \AA^{2} /$ molecule. The curves were fitted using eqs 1,3 , and 4 .

two cases. Thus, the SH signals then correspond to the $I_{1}$ values we want (Figure 5). The surface $\mathrm{p} K_{\mathrm{a}}$ values for them were $10.1 \pm 0.2$ and $10.2 \pm 0.2$, respectively. In our previous paper, without any of the considerations used above, the fitting result for the data with total density $30 \AA^{2} /$ molecule $\left(1 / \sigma_{\mathrm{m}}\right)$ gave a surface $\mathrm{p} K_{\mathrm{a}}=9.9 \pm 0.2$. We conclude that the closer the total surface density is to that of the coexistence to liquid turning point $S_{\text {l }}$, i.e., the homogeneous surface, the smaller is the need to make any corrections to the calculation of the surface $\mathrm{p} K_{\mathrm{a}}$ values. (a)

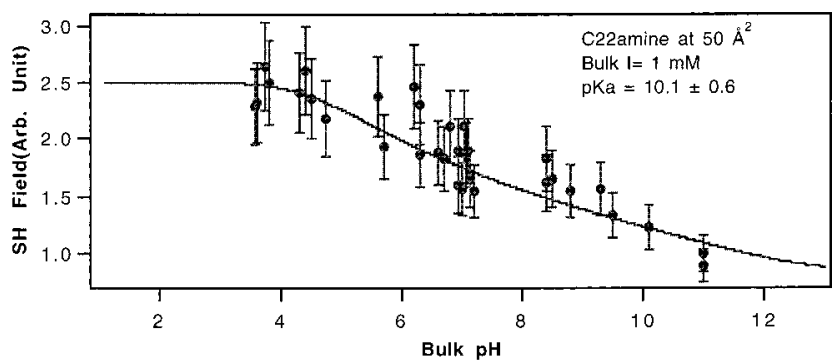

(b)

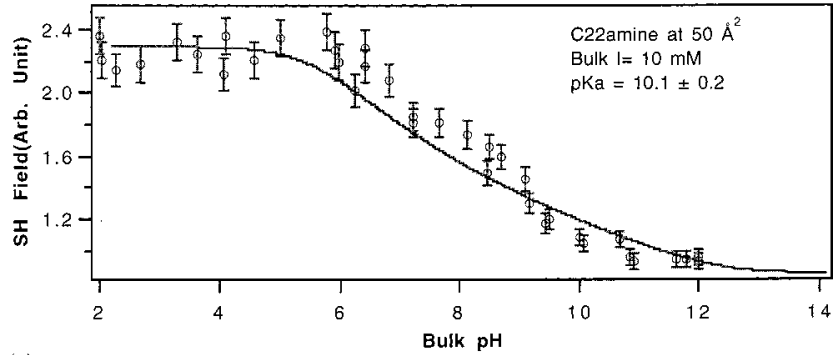

(c)

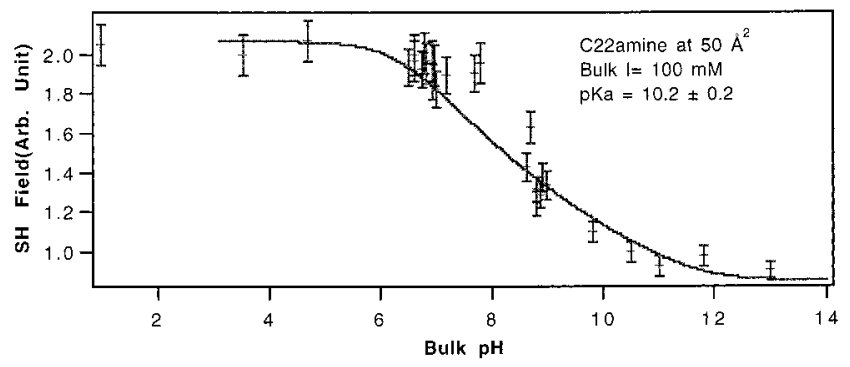

Figure 6. Calculated $\sqrt{\mathrm{I}_{1}}$ and fitting (solid line) for $50 \AA^{2} /$ molecule at different bulk electrolyte concentrations: (a) $1 \mathrm{mM}$; (b) $10 \mathrm{mM}$; (c) $100 \mathrm{mM}$. The curves were fitted using eqs 1,3 , and 4.

TABLE 2: Fitting Parameters and Results for Different Bulk Electrolyte Concentrations at a Surface Density of $50 \AA^{2} /$ molecule

\begin{tabular}{|c|c|c|c|}
\hline \multicolumn{2}{|c|}{$S_{1}=25 \AA^{2} /$ molecule } & \multicolumn{2}{|c|}{$S_{\mathrm{g}}=800 \AA^{2} /$ molecule } \\
\hline$I(\mathrm{mM})$ & $X_{1}$ & $1 / \sigma_{\mathrm{m}}\left(\AA^{2} /\right.$ molecule $)$ & surface $\mathrm{p} K_{\mathrm{a}}$ \\
\hline 1 & 0.625 & 32 & $10.1 \pm 0.6$ \\
\hline 10 & 0.484 & 25 & $10.1 \pm 0.2$ \\
\hline 100 & 0.484 & 25 & $10.2 \pm 0.2$ \\
\hline
\end{tabular}

4.2. Effect of Electrolyte on Surface $\mathbf{p} \boldsymbol{K}_{\mathrm{a}}$. Having established the treatment for obtaining the correct surface $\mathrm{p} K_{\mathrm{a}}$ at different surface densities, we now apply the same treatment to the effects of electrolyte on the interface acid-base equilibria for these systems.

Figure 6 and Table 2 show the experimental data for the $\mathrm{SH}$ field vs bulk $\mathrm{pH}$ for the $\mathrm{C}_{22}$ amine at a fixed average surface density $50 \AA^{2} /$ molecule for an electrolyte concentration of 1 , 10 , and $100 \mathrm{mM}$, respectively. We notice that the $\mathrm{SH}$ field in the low $\mathrm{pH}$ region decreases as the bulk electrolyte concentration increases from 1 to 10 to $100 \mathrm{mM}$ at the same bulk pH. This is because at the higher electrolyte concentrations the surface potential is smaller due to the screening of the surface charge by the electrolyte. Therefore, the contribution from the $\chi^{(3)}$ part to the $\mathrm{SH}$ field, which is proportional to the surface potential and in phase with the $\chi^{(2)}$ part, is smaller.

We notice in our experiment (Figure 6a) that the data for 1 $\mathrm{mM}$ show considerable scattering at $\mathrm{pH}$ values lower than 8 , whereas the data for 10 and $100 \mathrm{mM}$ do not. At low bulk electrolyte concentration, the repulsive interactions between charged amine headgroups are much larger, which further 
expand the liquid phase at the same surface charge density and therefore raise the surface pressure. If the surface pressure is too high (e.g., above equilibrium surface pressure), the instability of the insoluble monolayer can result in possible monolayer collapse $^{15}$ and therefore cause $\mathrm{SH}$ signal fluctuations. These observations are consistent with simulation results with continuum electrostatics model. ${ }^{17}$ The simulations predict no dependence of surface $\mathrm{p} K_{\mathrm{a}}$ of the $\mathrm{C}_{22}$ amine at the air/water interface on surface density and no dependence on bulk electrolyte concentration as low as $5 \mathrm{mM}$. But when the bulk electrolyte concentration is lower than $5 \mathrm{mM}$, the electrostatic energy of the monolayer becomes unstable.

Figure 6 also shows the calculated $\mathrm{SH}$ electric field of the liquid phase from eq 7 using the $\chi^{(3)}$ method described above. The $\mathrm{p} K_{\mathrm{a}}$ values we obtain at electrolyte concentrations in the stable electrolyte region are $10.1 \pm 0.6,10.1 \pm 0.2$, and $10.2 \pm 0.2$ for 1,10 , and $100 \mathrm{mM}$, respectively. The error bar for $1 \mathrm{mM}$ is large due to the $\mathrm{SHG}$ fluctuation at low $\mathrm{pH}$. Those $\mathrm{p} K_{\mathrm{a}}$ values indicate that there is no dependence of $\mathrm{p} K_{\mathrm{a}}$ value on the bulk electrolyte concentrations in the electrolyte range of $1-100 \mathrm{mM}$, which is in agreement with the orientational simulation. For typical weak organic acids and bases, the bulk $\mathrm{p} K_{\mathrm{a}}$ values will change no more than 0.2 units in the same electrolyte range of $1-100 \mathrm{mM} .^{22}$

4.3. General Discussion. It has been suggested ${ }^{18 a}$ that the shift of the surface $\mathrm{p} K_{\mathrm{a}}$ with respect to the bulk $\mathrm{p} K_{\mathrm{a}}$ value of $\mathrm{C}_{22}$ amine should not be very large; however, the basis for this suggestion was not provided. With certain assumptions ${ }^{15 \mathrm{c}}$ it is possible to estimate the ionization constants in the monolayer from surface pressure data ${ }^{18 \mathrm{~b}}$ or surface potential measurements. ${ }^{18 \mathrm{c}}$ With this latter approach results on the $n$-nonadecylamine $\left(\mathrm{C}_{19}\right.$ amine) monolayer at $A=25 \AA^{2} /$ molecule yielded a $\mathrm{p} K_{\mathrm{a}}$ of 10.1 at air/water interface, ${ }^{18 \mathrm{c}}$ which is clearly in excellent agreement with our SH results.

In the bulk solution hexyl, octyl and other small chain alkylamines have a bulk $\mathrm{p} K_{\mathrm{a}}$ of $10.6 .{ }^{19}$ Assuming that the bulk $\mathrm{p} K_{\mathrm{a}}$ of these shorter chain amines and docosylamine are the same we conclude that the $\mathrm{p} K_{\mathrm{a}}$ of $\mathrm{C}_{22}$ amine at the interface is lower than that in the bulk by 0.5 units, thus indicating that $\mathrm{C}_{22} \mathrm{NH}_{3}{ }^{+}$is more acidic by a factor of 3 at the air/water interface. The increased acidity is due to the higher interface free energy of the charged $\mathrm{C}_{22} \mathrm{NH}_{3}{ }^{+}$because of a decrease in solvent stabilization of the charged form at the interface relative to the bulk solution. The increased acidity is consistent with the studies of various adsorbates such as the long chain aniline, ${ }^{20}$ e.g., hexadecyl aniline $\left(\mathrm{C}_{16}\right.$ aniline $),{ }^{19}$ where it was found that the $\mathrm{p} K_{\mathrm{a}}$ of the anilinium group at the air/water interface is $3.6 \pm$ 0.3 , compared to a value of 5.3 in the bulk.

We know that the charged moiety in an acid-base reaction can be the acid or the base forms

$$
\begin{aligned}
& \mathrm{HA}^{+}+\mathrm{H}_{2} \mathrm{O} \rightleftharpoons \mathrm{A}+\mathrm{H}_{3} \mathrm{O}^{+} \\
& \mathrm{HA}+\mathrm{H}_{2} \mathrm{O} \rightleftharpoons \mathrm{A}^{-}+\mathrm{H}_{3} \mathrm{O}^{+}
\end{aligned}
$$

Aniline and amine are type I, while hydroxy acids such as phenol and nitrophenol are type II. It was found that for $p$-nitrophenol ${ }^{10}$ and $p$-octadecyl phenol $\left(\mathrm{C}_{18}\right.$ phenol $),{ }^{21}$ the acidity at the surface decreased, i.e., the surface $\mathrm{p} K_{\mathrm{a}}$ shifted to a higher value than in bulk. The surface $\mathrm{p} K_{\mathrm{a}}$ obtained for $p$-octadecyl phenol was $11.7 \pm 0.2^{21}$ compared with the bulk value 10.0. Type I and type II equilibria shift in opposite directions because the energetics at the air/water surface favors the neutral species.

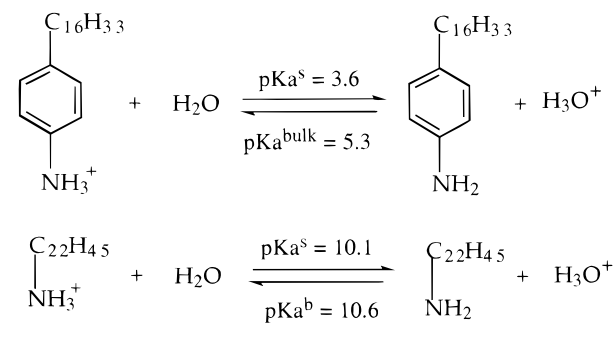

The shift of the surface $\mathrm{p} K_{\mathrm{a}}$ relative to the corresponding bulk $\mathrm{p} K_{\mathrm{a}}$ value of the $\mathrm{C}_{22}$ alkylamine is much smaller than that of $\mathrm{C}_{16}$ aniline (0.5 vs 1.7 units). We also note that the shift of $\mathrm{C}_{18}$ phenol is also much bigger than that of $\mathrm{C}_{22}$ amine (1.7 vs 0.5 ). One possibility is the effect of the location in the interface of the amine headgroup on the interface $\mathrm{p} K_{\mathrm{a}}$. The $\mathrm{C}_{22}$ amine might be expected to be deeper in the water than the aniline because the charge on the alkylamine is more localized, giving a larger electrostatic field. This deeper penetration into the bulk solution could make the environment of the $\mathrm{C}_{22}$ amine headgroup more closely resemble the environment in the bulk than would be the case for the $\mathrm{C}_{16}$ aniline headgroup.

\section{Conclusion}

Second harmonic generation was used to measure acid-base equilibria at the air/water interface and to investigate the dependence of the measured $\mathrm{p} K_{\mathrm{a}}$ value of the acid $\mathrm{CH}_{3}\left(\mathrm{CH}_{2}\right)_{21}$ $\mathrm{NH}_{3}{ }^{+}$on the surface monolayer density and bulk electrolyte concentration. The SHG signal depends linearly on the electrostatic potential at the surface, which in turn is dependent on the interface charge density, and thus the density of the interface acidic form $\mathrm{C}_{22} \mathrm{NH}_{3}{ }^{+}$. The Guoy-Chapman model was used to obtain the interface charge density from the surface potential determined from the SH measurements. Because the interface is inhomogeneous at low densities, consisting of "liquid" phase density $\mathrm{C}_{22}$ amine islands immersed in a gas phase of isolated "gas" phase $\mathrm{C}_{22}$ amine molecules, the Guoy-Chapman model, which assumes a uniform interface charge distribution, must be modified in its application to the inhomogeneous interface. If the surface density is assumed to be uniform, the application of the Guoy-Chapman model yields $\mathrm{p} K_{\mathrm{a}}$ values ranging from 8.2 to 10.2 as the density varies from 22 to $100 \AA^{2} /$ molecule. By accounting for the inhomogeneous surface, a single $\mathrm{p} K_{\mathrm{a}}$ value of $10.1 \pm 0.2$ is obtained at all densities. Thus, we conclude that there is no significant dependence of $\mathrm{p} K_{\mathrm{a}}$ on surface monolayer density. The bulk $\mathrm{p} K_{\mathrm{a}}$ value of the long chain alkylamines is 10.6 . This increased surface acidity by a factor of 3 above its bulk value is due to the higher free energy of a charged species at the air/water interface than in the bulk solution. The acid-base equilibrium is shifted to the energetically more favorable neutral form at the interface. With respect to any $\mathrm{p} K_{\mathrm{a}}$ dependence on electrolyte concentration, we found none over a wide range extending from a bulk electrolyte from 1 to $100 \mathrm{mM}$. At a $1 \mathrm{mM}$ electrolyte concentration we observe fluctuations in the $\mathrm{SH}$ signal, which we attribute to the importance of the increase in charge-charge repulsive interactions at this low electrolyte density. These fluctuations reflect the instability in liquid island sizes, shapes, and possible monolayer collapse. These observations are supported by simulation studies. ${ }^{17}$

Acknowledgment. We thank the Division of Chemical Science of the Department of Energy and the National Science Foundation for their support and Yan Liu for her assistance. 


\section{References and Notes}

(1) Adamson, A. W. Physical Chemistry of Surfaces, 5th ed.; John Wiley \& Sons: New York, 1990

(2) (a) Eisenthal, K. B. Annu. Rev. Phys. Chem. 1992, 43, 627. (b)

Corn, R. M.; Higgins, D. A. Chem. Rev. 1994, 94, 107-25.

(3) Bloembergen, N.; Pershan, P. S. Phys. Rev. 1962, 128, 606.

(4) Shen, Y. R. The Principles of Nonlinear Optics; Wiley: New York, 1984.

(5) Chen, C. K.; Heinz, T. F.; Ricard, D.; Shen, Y. R. Phys. Rev. Lett.

1981, 46, 146; Chem. Phys. Lett. 1981, 83, 455; Phys. Rev. 1983, B27, 1965.

(6) (a) Hicks, J. M.; Kemnitz, K.; Eisenthal, K. B.; Heinz, T. F. J.

Phys. Chem. 1986, 90, 560. (b) Kemnitz, K.; Bhattacharyya, K.; Pinto, G. R.; Eisenthal, K. B.; Heinz, T. F. Chem. Phys. Lett. 1986, 131, 285.

(7) Harris, A. L.; Chidsey, C. E. D.; Levinos, N. J.; Loiacone, D. N. Chem. Phys. Lett. 1987, 141, 350.

(8) Richmond, G.; Robinson, J. M.; Shannon, V. L. Prog. Surf. Sci. 1980, 28,1 .

(9) (a) Xiao, X. D.; Vogel, V.; Shen, Y. R. Chem. Phys. Lett. 1989, 163, 555. (b) Zhao, X.; Subrahmanyan, S.; Eisenthal, K. B. Chem. Phys. Lett. 1990, 171, 558.

(10) Bhattacharyya, K.; Sitzmann, E. V.; Eisenthal, K. B. J. Chem. Phys. 1987, 87, 1442. 327.
(12) Zhao, X.; Ong, S.; Eisenthal, K. B. Chem. Phys. Lett. 1993, 202, 513.

(13) Zhao, X.; Ong, S.; Wang, H.; Eisenthal, K. B. Chem. Phys. Lett. 1993, 214, 208.

(14) McLaughlin, S. Annu. Rev. Biophys. Biophys. Chem. 1989, 18, $113-36$.

(15) Gaines, G. L., Jr. Insoluble Monolayers at Liquid-Gas Interfaces; Wiley: New York, 1965; (a) p 159, (b) p 188, (c) p 233.

(16) (a) Zhao, X.; Goh, M. C.: Subrahmanyan, S.; Eisenthal, K. B. J Phys. Chem. 1990, 94, 3370. (b) Xiaolin Zhao Ph.D. Thesis, Department of Chemistry, Columbia University, 1992.

(17) Smart, J. L.; McCammon, J. A. J. Am. Chem. Soc. 1996, 118 $2283-4$.

(18) (a) Lu, J. R.; Simister, E. A.; Lee, E. M.; Thomas, R. K.; Rennie, A. R.; Penfold, J. Langmuir 1992, 8, 1837. (b) Liu, J. R.; Li, Z. X.; Smallwood, J.; Thomas, R. K.; Penfold, J. J. Phys. Chem. 1995, 99, 823343.

(19) CRC Handbook of Chemistry and Physics; CRC Press: Cleveland, OH, 1974; D147-D149.

(20) Castro, A.; Bhattacharyya, K.; Eisenthal, K. B. J. Chem. Phys. 1991, 95, 1310.

(21) Subrahmanyan, S.; Zhao, X. L.; Eisenthal, K. B. To be submitted. (22) See for example, Harned, H. S.; Owen, B. B. The Physical Chemistry of Electrolytic Solutions, 3rd ed.; Reinhold Publishing Corp. New York, 1958; pp 669-81. 